\title{
Archéopages
}

Archéopages

Archéologie et société

40 | 04-07/2014

Villages

\section{État de la recherche sur le « fait villageois » en Picardie méridionale. Nouvelles données et nouvelles perspectives}

Louis Hugonnier, Stéphane Arbault, Martine Derbois, Gilles Desplanque, Richard Fronty, Denis Maréchal, Sylvain Rassat et Sébastien Ziegler

\section{(2) OpenEdition \\ Journals}

Édition électronique

URL : https://journals.openedition.org/archeopages/599

DOI : 10.4000/archeopages.599

ISSN : 2269-9872

Éditeur

INRAP - Institut national de recherches archéologiques préventives

Édition imprimée

Date de publication : 1 juin 2015

Pagination : $56-63$

ISSN : 1622-8545

Référence électronique

Louis Hugonnier, Stéphane Arbault, Martine Derbois, Gilles Desplanque, Richard Fronty, Denis Maréchal, Sylvain Rassat et Sébastien Ziegler, «État de la recherche sur le « fait villageois » en Picardie méridionale. Nouvelles données et nouvelles perspectives », Archéopages [En ligne], 40 | 04-07/2014, mis en ligne le 01 juillet 2016, consulté le 22 janvier 2022. URL : http:// journals.openedition.org/archeopages/599; DOI : https://doi.org/10.4000/archeopages.599 


\title{
État de la recherche sur le « fait
}

\section{villageois » en Picardie méridionale Nouvelles données et nouvelles perspectives}

\author{
Louis Hugonnier Inrap \\ Stéphane Arbault Ministere de la culture et del la Communication \\ Martine Derbois Inrap \\ Gilles Desplanque Département de latisne \\ Richard Fronty Inrap

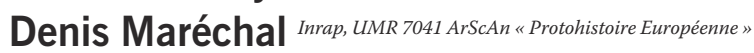 \\ Sylvain Rassat Ministere de la cultureet de la Communication \\ avec la collaboration de Hélène Dulauroy-Lynch et Danaël Veyssier Conseilgenteralde roise \\ Sébastien Ziegler villede Chateau-Thierry
}

\section{6}

1. L'ARC n ${ }^{\circ} 4420$

« Habitats et territoires, occupations médiévales, Aisne-Oise », intégré à la thématique de l'axe de recherche Inrap 2010-7 Milieux ruraux et urbains au Moyen Âge et époque Moderne, regroupe différents acteurs régionaux (SRA Picardie, Inrap Picardie, Conseil général de l'Aisne, Conseil général de l'Oise, Service archéologique de la Ville de Noyon, Unité d'archéologie de Château-Thierry) dont les champs d'actions recouvrent, comme le suggère l'intitulé de l'axe, les thématiques liées aux différentes formes d'habitats médiévaux et les relations de ces derniers avec leurs territoires.

2. La liste n'est malheureusement pas exhaustive, la dynamique ayant à gérer notamment la difficulté d'accès à un certain nombre d'opérations. On gardera également à l'esprit le caractère post-fouille en cours de $80 \%$ de ces gisements.
Les principaux constats soulignés dans l'articlebilan sur « les campagnes médiévales en Picardie: État de la question » publié par la Revue archéologique de Picardie (Blary et al., 2005) faisaient état d'un manque cruel de synthèses sur l'habitat rural et le «fait villageois », de sa plus simple expression à sa complexité environnementale. Il pointait du doigt le manque de synergie dans la recherche, la dichotomie entre le nombre de faits observés et celui des faits publiés, la faiblesse des effectifs d'intervenants médiévistes et des moyens consacrés aux études. Cet article interpellait le lecteur sur l'absence de participation des archéologues régionaux sur la question relative à la « naissance du village». Une des conclusions soulignait « le manque de programmes collectifs » alors que « la documentation est abondante, de grande qualité, mais d'accès difficile » (ibid., p. 237-238). La hausse des effectifs d'archéologues médiévistes ces dernières années au sein des équipes des acteurs régionaux de l'archéologie préventive et les récentes politiques d'aménagement des périphéries proches et centresbourgs actuels ont fourni l'opportunité de constituer en janvier 2014, un axe de recherche collectif (ARC) intitulé « Habitats et territoires, occupations médiévales, Aisne-Oise » ${ }^{\mathbf{1}}$, dont l'une des problématiques cible le concept « village ». L'équipe recense actuellement les données nouvelles (les opérations entre 2006 et 2014), pour les intégrer dans une base de données générale où figure déjà une partie des données d'avant 2005.

\section{Bases de données et corpus 2006-2014}

Létablissement de la base de données se fait en étroite collaboration avec le SRA Picardie. Cette base interrelationnelle de données permet de croiser les informations issues de trois sources complémentaires. La première est la base de données nationale Patriarche qui regroupe toutes les informations issues des opérations archéologiques et les organise à l'aide d'un thésaurus commun et d'une indexation numérique des opérations et des faits archéologiques. La deuxième a été développée par le Service régional de l'archéologie de Picardie pour être dédiée spécifiquement à l'enregistrement des rapports scientifiques picards. La troisième et dernière base de données est, quant à elle, propre à l'ARC. Elle recueille le travail de synthèse de tous les membres du groupe de recherche et les données des deux bases précédemment citées. Elle a été établie à partir de l'ossature de la base de données de recherches de Martine Derbois. Le leitmotiv était de mettre en place un outil simple et efficace qui utilise, comme élément central, l'indexation de la base Patriarche. Cette structuration permet d'obtenir une échelle d'étude intra site et régionale, tout en évacuant au maximum les problèmes liés à l'hétérogénéité et à la qualification de la donnée archéologique. De plus, ce travail en amont autorise la déclinaison des résultats statistiques et cartographiques selon des problématiques précises telle que celle qui nous rassemble ici : 128 opérations menées dans l'Oise et l'Aisne entre 2005 et 2014 relèvent ainsi des problématiques liées à la « naissance du village » [ill. 1]. Les données sont reprises dans un dernier temps par les archéologues, qui effectuent un nouveau dépouillement des informations archéologiques au vu de leur connaissance du terrain et leur maîtrise bibliographique.

À nouveaux aménagements, nouvelles données

Le présent corpus [ill. 2 et 3] et concerne 31 occurrences $^{2}$ relevées entre 2005 et 2014 ( 21 fouilles et 10 diagnostics). La surface d'ouverture moyenne par site est de $4.451 \mathrm{~m}^{2}$ et d'environ un demi- 

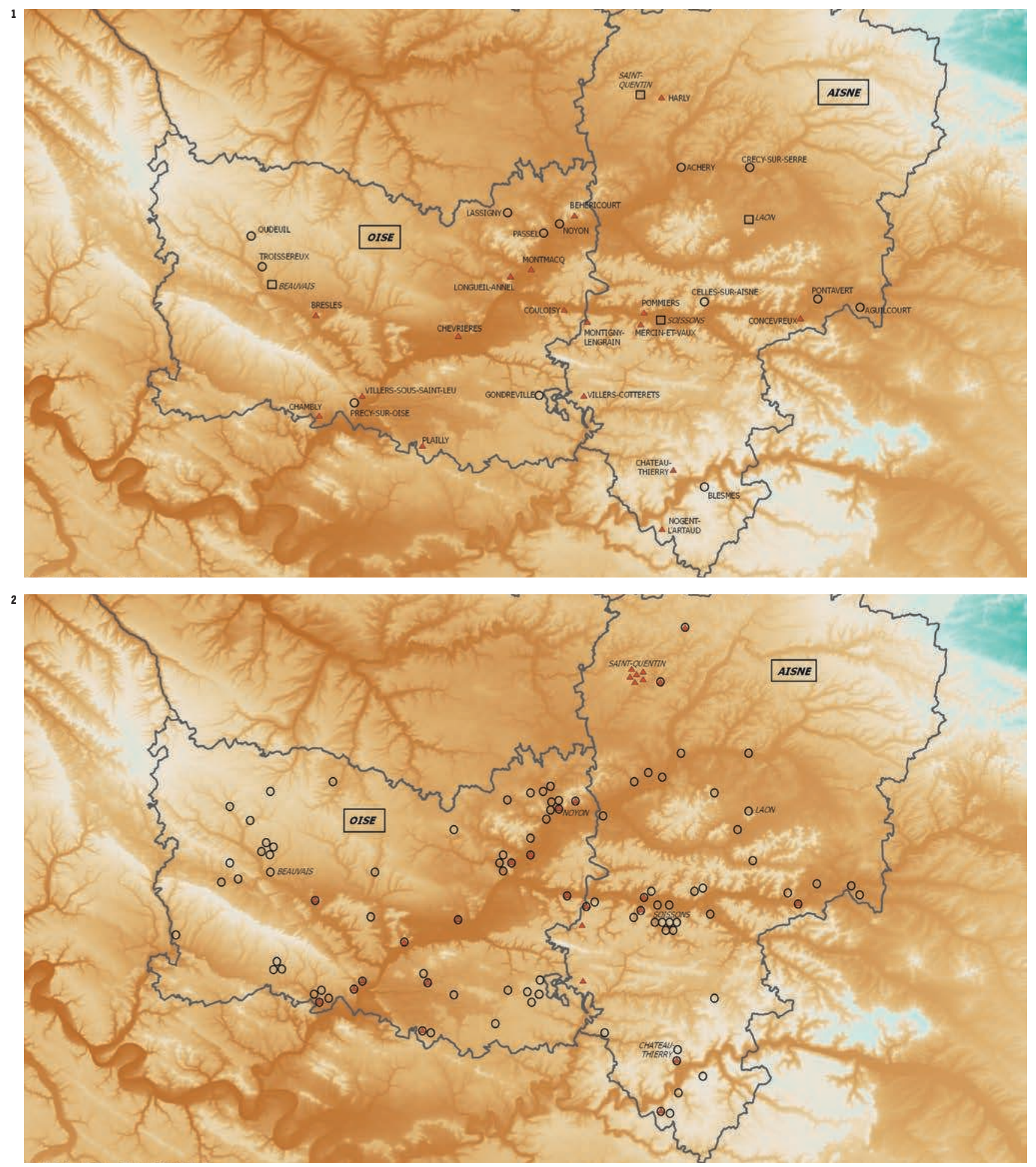

1.Opérations menées entre 2005 et 2014 ayant

livré des vestiges liés à la question de la naissance du village.

2. Les 31 sites qui forment

le corpus de cet article

constituent une surface

totale d'ouverture

avoisinant les 13,8 ha

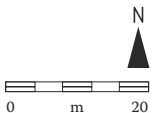

— Limites administratives

ㅁ Ville repère

Cas d'étude:

A Fouille archéoplogique

- Diagnostics archéologiques 


\begin{tabular}{|c|c|c|c|c|c|c|c|c|}
\hline $\mathrm{n}^{\circ}$ site & Toponyme du site & Opérateur & Année & Dpt & Commune & $\begin{array}{l}\text { Type inter- } \\
\text { vention }\end{array}$ & $\begin{array}{l}\text { Surface } \\
\text { ouverte }\end{array}$ & $\begin{array}{c}\text { Position / } \\
\text { centre bourg }\end{array}$ \\
\hline 1 & La Croix noire & INRAP & 2012 & Oise & Montmacq & $\mathrm{F}$ & 1ha & $<100 \mathrm{~m}$ \\
\hline 2 & Le Chemin du châtelain & NOYON & 2006 & Oise & Noyon & $\mathrm{D}$ & $1581 \mathrm{~m}^{2}$ & extérieur au faubourg \\
\hline 3 & ZA Noyon-Passel 2007 & NOYON & 2007 & Oise & Passel & $\mathrm{F}$ & $5945 \mathrm{~m}^{2}$ & $500 \mathrm{~m}$ \\
\hline 4 & $\begin{array}{l}\text { Le Fumeron place } \\
\text { publique }\end{array}$ & NOYON & 2011 & Oise & Béhéricourt & $\mathrm{F}$ & $1490 \mathrm{~m}^{2}$ & bordure du château \\
\hline 5 & Les Quatre livres & CG02 & 2013 & Aisne & Mercin-et-Vaux & $\mathrm{F}$ & $3000 \mathrm{~m}^{2}$ & périph.église \\
\hline 6 & Les Grosses vignes & CG02 & 2012 & Aisne & Pommiers & $\mathrm{F}$ & $3000 \mathrm{~m}^{2}$ & périph.église \\
\hline 7 & 7 rue Porteneuve & UACT & 2009 & Aisne & Nogent l'Artaud & $\mathrm{F}$ & $1600 \mathrm{~m}^{2}$ & périph.village méd \\
\hline 8 & 3-5 rue de la Madelaine & UACT & 2010 & Aisne & Château-thierry & $\mathrm{F}$ & $2000 \mathrm{~m}^{2}$ & cœur ville \\
\hline 9 & Derrière Jardiland, Rn3 & UACT & 2006 & Aisne & Blesmes & $\mathrm{D}$ & 2 ha & périph.village méd \\
\hline 10 & Le Village & INRAP & 2010 & Oise & Couloisy & $\mathrm{F}$ & $8000 \mathrm{~m}^{2}$ & centre \\
\hline 11 & 803 rue Henri Barbusse & INRAP & 2011 & Oise & Chambly & $\mathrm{F}$ & $3800 \mathrm{~m}^{2}$ & faubourg \\
\hline 12 & $13-21$ rue de Paris & CG60 & 2011 & Oise & Plailly & $\mathrm{F}$ & $1200 \mathrm{~m}^{2}$ & centre bourg \\
\hline 13 & rue de Boissy site 1 & INRAP & 2009 & Oise & Villers-sous-st-leu & $\mathrm{F}$ & $6000 \mathrm{~m}^{2}$ & centre bourg \\
\hline 14 & rue de Boissy site 2 & INRAP & 2009 & Oise & Villers-sous-st-leu & $\mathrm{F}$ & $4000 \mathrm{~m}^{2}$ & centre bourg \\
\hline 15 & rue du Martray & INRAP & 2013 & Oise & précy-sur-Oise & $\mathrm{F}$ & $5400 \mathrm{~m}^{2}$ & périphérie \\
\hline 16 & Le Babouin - RD 234 & CG60 & 2011 & Aisne & Bresles & $\mathrm{F}$ & $2000 \mathrm{~m}^{2}$ & \\
\hline 17 & La Braze & CG60 & 2013 & Oise & Gondreville & $\mathrm{D}$ & $15000 \mathrm{~m}^{2}$ & $600 \mathrm{~m}$ \\
\hline 18 & rue saint Omer (mairie) & INRAP & 2012 & Oise & Oudeuil & $\mathrm{D}$ & $182 \mathrm{~m}^{2}$ & proche église romane \\
\hline 19 & 7, 9 et 11 r. Gal Patton & INRAP & 2013 & Aisne & Crécy-sur-Serre & $\mathrm{D}$ & $80-630 \mathrm{~m}^{2}$ & centre \\
\hline 20 & 25-27 rue de Calais & INRAP & 2013 & Oise & Troissereux & $\mathrm{D}$ & $4567 \mathrm{~m}^{2}$ & périph.directe \\
\hline 21 & Rue du Fond Talon & INRAP & $2010-14$ & Aisne & $\begin{array}{l}\text { Montigny-Len- } \\
\text { grain }\end{array}$ & $\mathrm{F}$ & $800 \mathrm{~m}^{2}$ & $\begin{array}{l}\text { hameau } \\
\text { (cf.église) }\end{array}$ \\
\hline 22 & Rue de la Marne & INRAP & 2013 & Aisne & Harly & $\mathrm{F}$ & $1500 \mathrm{~m}^{2}$ & $100 \mathrm{~m}$ de l'église \\
\hline 23 & Le petit Château & INRAP & 2007 & Aisne & Aguilcourt & $\mathrm{F}$ & $1180 \mathrm{~m}^{2}$ & périphérie du village \\
\hline 24 & Le Village & INRAP & 2011 & Oise & Longueil-Annel & $\mathrm{F}$ & $15888 \mathrm{~m}^{2}$ & $\begin{array}{l}\text { périphérie nord } \\
\text { du bourg }\end{array}$ \\
\hline 25 & le Poirier d'Oignon & INRAP & 2012 & Aisne & Villers-Cotterêts & $\mathrm{F}$ & $23600 \mathrm{~m}^{2}$ & $\begin{array}{l}\text { périphérie sud } \\
\text { de la commune }\end{array}$ \\
\hline 26 & le Grand Routy & INRAP & 2012 & Aisne & Concevreux & $\mathrm{F}$ & $1200 \mathrm{~m}^{2}$ & $\begin{array}{c}\text { périphérie du bourg } \\
\text { actuel }\end{array}$ \\
\hline 27 & rue de la Misacart & INRAP & 2010 & Oise & Lassigny & $\mathrm{D}$ & $726 \mathrm{~m}^{2}$ & $\begin{array}{c}\text { périphérie du bourg } \\
\text { actuel }\end{array}$ \\
\hline 28 & rue Charles de Gaulle & INRAP & 2012 & Oise & Achery & $\mathrm{D}$ & $179 \mathrm{~m}^{2}$ & centre bourg $250 \mathrm{~m}$ \\
\hline 29 & route de Soissons & INRAP & 2012 & Aisne & Pontavert & $\mathrm{D}$ & $26945 \mathrm{~m}^{2}$ & périphérie \\
\hline 30 & Chemin de la patte d'oie & INRAP & 2008 & Aisne & Celles sur Aisne & $\mathrm{D}$ & $7168 \mathrm{~m}^{2}$ & extérieur du bourg \\
\hline 31 & Le Village & INRAP & 2014 & Oise & Chevrières & $\mathrm{F}$ & $5300 \mathrm{~m}^{2}$ & $80 \mathrm{~m}$ centre bourg \\
\hline
\end{tabular}


3. Sur les 2325 opérations archéologiques (dont

425 fouilles) ayant eu lieu dans l'Oise et l'Aisne entre 2005 et 2014, on relève que 128 opérations - soit $0,05 \%$ - (dont 31 fouilles soit $0,07 \%$ ) ont livré des vestiges archéologiques témoignant des problématiques liées à la « naissance du village ». Les deux départements où ont été choisis les sites étudiés sont marqués historiquement par les occupations des vallées de l'Oise et de l'Aisne.
Emprise du bourg actuel Nécropole $\mathrm{VI}^{\mathrm{e}}-\mathrm{IX}^{\mathrm{e}}$ siècle Unités domestiques Voierie ancienne Sarcophages mis au jour en 1951 Sondages archéologiques

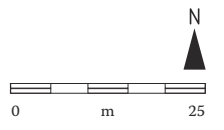

hectare $\left(5040 \mathrm{~m}^{2}\right)$ si l'on fait abstraction des diagnostics. On est bien loin des hectares franciliens (pour exemple Serris, « les Ruelles », $16 \mathrm{ha}$ ) et de ce besoin de grandes surfaces pour appréhender le phénomène village dans toutes ses composantes (Blaising, Gérard, 2006 ; Gentili, 2010). Si la surface relativement réduite des opérations du corpus restreint la vision, la topographie des récentes interventions permet de porter un nouveau regard sur le phénomène : 13 opérations ont eu lieu à proximité directe de l'église (entre o et $100 \mathrm{~m}), 11$ en périphérie directe du village $(100<500 \mathrm{~m})$, quatre dans les faubourgs et trois en périphérie lointaine. Ce « recentrage » pour les aménageurs est une véritable aubaine pour les archéologues : il est dès lors possible de fouiller au cœur du village (Valais, 2012). Quand bien même les surfaces sont restreintes dans ces cœurs de village, et même si les diagnostics positifs n'aboutissent pas obligatoirement à une prescription de fouille, les informations récoltées sont fondamentales : le gisement de la « Rue SaintOmer » à Oudeuil ( $\left.\mathrm{n}^{\circ} 18\right)$ est l'exemple récent le plus marquant (Fronty et al., 2013) [ill. 4]. Cette opération a mis au jour, parmi une cinquantaine de structures et horizons anthropiques, une nécropole du haut Moyen Âge. La nécropole présente une forte densité de sépultures avec de fréquents recoupements. Les tombes disposées régulièrement ont quatre orientations distinctes et sont datables, au vu du mobilier récolté, entre la fin du VI ${ }^{\mathrm{e}}$-début $\mathrm{VII}^{\mathrm{e}}$ siècle et le VIII ${ }^{\mathrm{e}}$ - $\mathrm{IX}^{\mathrm{e}}$ siècle. On a un aperçu, malgré la faible densité d'ouverture, de l'organisation et de la structuration de la nécropole : absence de sépultures dans le sondage 3 et présence de plusieurs bâtiments, matérialisés par l'existence de vingt-trois trous de poteaux et de fosses, situés au sud de la zone funéraire. Cette opération, malgré sa surface d'ouverture limitée $\left(182 \mathrm{~m}^{2}\right)$, révèle ainsi la présence du cimetière alto-médiéval au centre du village actuel, associé à une occupation domestique contemporaine de ce lieu de sépulture, association courante au VII ${ }^{\mathrm{e}}$ siècle (Lorren, Périn, 1995 ; Martin et al., 2011). L'inventeur du gisement,
Richard Fronty, suggère, « concernant la question de l'origine et des modalités de fixation, que chapelle et cimetière peuvent être ici à l'origine du village d'Oudeuil, même s'il est difficile d'en préciser la nature et que le processus peut s'étaler sur une longue période » (Fronty, 2013 ; Treffort, 1996). Dans un contexte identique d'intervention, le gisement des « Quatre Livres » à Mercin-et-Vaux $\left(n^{\circ} 5\right)$ a livré une vision plus large, de par sa surface $\left(3000 \mathrm{~m}^{2}\right)$ et sa nature. La fouille a notamment mis en évidence une occupation domestique altomédiévale, $30 \mathrm{~m}$ à l'est de l'église romane. Pour la période mérovingienne, l'occupation (cabanes excavées, bâtiments sur poteaux) est circonscrite au sein de plusieurs enclos quadrangulaires perpendiculaires à la pente. Dès le VIII ${ }^{\mathrm{e}}$ siècle, l'occupation se recentre vers le haut de la parcelle, formant alors une couronne a priori centrée sur l'espace occupé actuellement par l'église. Dans le courant du $\mathrm{x}^{\mathrm{e}}$ siècle, un vaste bâtiment excavé $\left(75 \mathrm{~m}^{2}\right)$ aux parois maçonnées prend place, unique témoin de cette phase d'occupation. L'abandon semble se situer entre le $\mathrm{XI}^{\mathrm{e}}$ et le $\mathrm{XII}^{\mathrm{e}}$ siècle (séquence de colluvionnement). Une partie du cimetière paroissial médiéval attenante à l'église actuelle, a également été mise au jour, et près de 90 inhumations attribuées à une période comprise entre les $\mathrm{IX}^{\mathrm{e}}$ et $\mathrm{XII}^{\mathrm{e}}$ siècles ont été fouillées. L'abandon semble se situer au plus tard au XIII ${ }^{\mathrm{e}}$ siècle. Gilles Desplanque, responsable de l'opération, précise que « si les datations des sépultures se confirment, l'évidence d'une simultanéité entre l'occupation funéraire et les occupations domestiques pour la seconde moitié de la période alto-médiévale serait établie », simultanéité faisant écho à celle d'Oudeuil (Desplanque, 2012). Ce premier regard peut alors s'étendre sur les 31 sites du corpus 2006-2014, afin de percevoir le potentiel existant et mis au jour ces huit dernières années. On pourra alors, dans un premier temps, se référer et se confronter aux conclusions établies par Édith Peytremann, notamment en ce qui concerne la périodisation de ces habitats (Peytremann, 2003, et article dans ce numéro). 


\section{Des caractéristiques d'implantations}

$60 \%$ des gisements du corpus sont situés dans des contextes de versants et de fonds de vallée (19 sites sur 31), la moitié des 12 restants étant localisée sur les rebords de plateaux. Cette prééminence des zones de vallées a déjà été signalée pour les installations des $\mathrm{V}^{\mathrm{e}}-\mathrm{XV}^{\mathrm{e}}$ siècles (Peytremann, 2003). Par ailleurs, cette proximité avec l'eau est récurrente dans l'ensemble des sites, qui sont tous installés à moins de $500 \mathrm{~m}$ de l'eau et majoritairement à une distance comprise entre o et $100 \mathrm{~m}$ - sachant que la distance o-5o m concerne la présence sur le site soit d'un ru (les rus Cordier et Mannier à Couloisy [ $\mathrm{n}^{\circ} 10$ ] traversant le centre du village) ou de puits (16 puits ont été pour le moment recensés et identifiés sur les 31 sites). Une exception figure à la règle, celle $\mathrm{du}$ « Fumeron place publique » à Béhéricourt $\left(\mathrm{n}^{\circ} 4\right)$ où le seul point d'eau identifié correspond à un abreuvoir. Mais on relativisera cette exception : le lieu étudié correspond à la périphérie haute de l'occupation alto-médiévale localisée vers la rupture entre le versant et la terrasse alluviale et l'accès à l'eau reste à identifier. Ce rapport à l'eau se retrouve également dans les contextes environnementaux de ces gisements puisque près des deux tiers sont localisés dans des milieux humides. Dans le cas de Couloisy ( $\left.{ }^{\circ} 10\right)$, les différentes occupations qui se sont succédé (Protohistoire à histoire moderne) ont soit généré des actions servant à maittriser ce milieu humide (fossé de drainage, vivier), soit subi le contexte (déplacement supposé de l'habitat du haut Moyen Âge hors de l'emprise des crues de l'ancien bras de l'Aisne identifié sur le territoire). Autre donnée d'implantation, celle concernant l'existence ou non de traces d'occupations antérieures et leur possible influence sur la structuration des habitats médiévaux. Ces traces sont quasi systématiques dans notre corpus, sur site ou en périphérie. Seuls les sites $\mathrm{du}$ "Chemin du Châtelain » $\left(\mathrm{n}^{\circ}{ }_{2}\right)$ et de « la Braze » $\left(n^{\circ} 17\right)$ semblent vierges de traces d'occupation anciennes. Celles-ci sont majoritairement liées à une occupation protohistorique (oppidum de Pommiers pour le site des « Grosses Vignes » $\left[\mathrm{n}^{\circ} 6\right]$ et de Bailleul sur Thérain pour « le Babouin - RD 234 » $\left[\mathrm{n}^{\circ} 16\right]$; enclos et/ou parcellaires à Couloisy $\left[\mathrm{n}^{\circ} 10\right]$ ) ou gallo-romaine (villae de Montmacq $\left[n^{\circ} 1\right]$, Mercin-et-Vaux [ $\left.n^{\circ} 5\right]$, Blesmes [ $\left.\left.n^{\circ} 9\right]\right)$. Au moins un tiers se situent sur ou à proximité directe d'une villa identifiée, la proportion étant quasi identique à celle de la présence d'une voie antique, l'association étant quasi systématique. Le gisement qui, à ce stade de l'analyse, connaît l'anthropisation la plus marquée au niveau de son territoire direct est « la rue du Martray » à Précy-sur-Oise ( $\left.\mathrm{n}^{\circ} 15\right)$ : occupation néolithique (fosses d'extractions), dépôt d'objets métalliques du Bronze final III, fossé Hallstatt, tombe gallo-romaine en sarcophage composent l'environnement direct du site. On ne peut encore se prononcer réellement sur l'influence des éléments anciens sur la structuration des habitats médiévaux concernés, au mieux on admettra que ces habitats pérennisent l'anthropisation des territoires concernés. Coïncidence ou influence, on peut évoquer le cas du fossé gallo-romain de Montmacq (Pissot et al., 2013, p. 66-67) dont le rôle de limite parcellaire dans le paysage antique a pu avoir une influence sur la structuration de l'habitat alto-médiéval identifié, celui-ci reproduisant les directions orthonormées de l'occupation gallo-romaine. Ces quelques caractéristiques mises en lumière au niveau des récentes opérations font écho, dans une certaine mesure, aux hypothèses avancées dès les années 1980 par Didier Bayard - relatives à l'« intensification de l'occupation des vallées et des vallons au détriment des sites de plateaux » (Bayard, 1986) - et aux données d'Édith Peytremann (Peytremann, 2003) - les sites de l'Aisne et de l'Oise qu'elle a recensés avant 2005 sont majoritairement installés dans les vallées -, ces deux auteurs insistant sur l'omniprésence des caractères hydrographiques de ces occupations ainsi que sur l'importance relative du substrat antique. Reste qu'il faut toujours garder à l'esprit l'effet de sources parfois pernicieux provoqué par la localisation topographique des aménagements.

\section{De la topographie des structures}

Dans la moitié des sites du corpus au moins, on note la présence d'éléments fossoyés participant de près ou de loin à la structuration de l'espace d'occupation : sept gisements ont au moins un chemin identifié sur le site et sept autres un réseau viaire attesté soit dans les sources écrites, soit en liaison avec un système antique. Dans une certaine mesure, certains gisements offrent, en outre, l'expression d'une possible influence de ces réseaux viaires sur l'agencement interne des habitats. C'est notamment le cas de Chevrières $\left(n^{\circ} 31\right)$ où le chemin creux mis au jour sert d'axe directeur [cf. encadré p. 64]. Les fossés identifiés concourent également pour certains à la séparation des espaces internes (Pommiers [ $\left.{ }^{\circ} 6\right]$, Mercin-et-Vaux [n $\left.{ }^{\circ} 5\right]$, Plailly [ $\left.{ }^{\circ} 12\right]$ ). En revanche, l'absence de fossé met en lumière un second type de partition de l'espace, fondée sur la corrélation entre chemin et espaces de concentration de vestiges, cernés ou non par des clôtures en bois ou en haies (Montmacq [ $\left.\mathrm{n}^{\circ} 1\right]$, Chevrières [ $\mathrm{n}^{\circ}{ }_{31}$ ], Villers-sous-Saint-Leu [ $\left.\mathrm{n}^{\circ} 13\right]$ ). Ainsi, la présence de fossés n'est pas la condition sine qua non de structuration interne d'un site. On note enfin le passage d'un système à l'autre à Longueil-Annel ( $\left.n^{\circ} 24\right)$. Pour ce qui est des bâtiments composant ces implantations, les gisements offrent un potentiel de synthèse non négligeable avec le recensement de 144 structures excavées de type «fonds de cabane » et, dans une moindre mesure, cave ou cellier ainsi qu'une soixantaine de bâtiments sur poteaux et/ou sablières. Trois sites se distinguent par leurs densités de vestiges : Montmacq $\left(\mathrm{n}^{\circ} 1-47\right.$ fonds de cabane, au moins 7 bâtiments sur poteaux), Villers-sous-Saint-Leu $\left(\mathrm{n}^{\circ} 13-30\right.$ fonds de cabane et une vingtaine de bâtiment sur poteaux) et 
Précy-sur-Oise $\left(\mathrm{n}^{\circ} 15-9\right.$ fonds de cabane et au moins 15 bâtiments sur poteaux). En exceptant les données des diagnostics, le nombre moyen par site de ces édifices reste inférieur à dix. La typologie de ces constructions n'ayant pas encore été établie, on ne peut qu'en souligner le potentiel ${ }^{3}$. Ce même potentiel se retrouve dans le recensement des structures dites « domestiques » comme les fours (57 identifiés) et les silos (environ 177). Là encore, certains sites se distinguent par la densité de ces structures : Villers-sous-Saint-Leu ( $\mathrm{n}^{\circ} 13$ - 30 silos identifiés), Concevreux (n 26 - 20 silos identifiés), mais surtout récemment Chevrières où s'égrènent sur le site 74, silos qui ont donné lieu par ailleurs à un protocole de prélèvements conséquent. Sur les 12 gisements où l'identification a pu être clarifiée, une nette prédominance de l'habitat groupé semble se mettre en place au cours de la période carolingienne, faisant suite à une occupation plus ou moins lâche à la période mérovingienne, dynamique avancée par Édith Peytremann dans notre secteur (Peytremann, 2003) et dont le meilleur exemple s'observe à Pommiers [cf. encadré p. 66]. Édith Peytremann relevait également un autre facteur intéressant sur huit sites de son corpus concernant la présence des morts dans l'espace ou le monde des vivants. Dans notre corpus, variables en nombre comme en distribution spatiale, des sépultures sont attestées sur dix des 31 sites intégrés, soit un tiers des gisements. Six gisements ont livré uniquement des tombes dispersées (Montmacq $\left[n^{\circ} 1\right]$, Pommiers [n $\left.{ }^{\circ} 6\right]$, Couloisy [ $\left.n^{\circ} 10\right]$, Achery [ $\left.n^{\circ} 28\right]$, Plailly [ $\left.n^{\circ} 12\right]$, Chevrières [ $\left.\left.n^{\circ} 31\right]\right)$ dont les dispositifs sépulcraux diffèrent : à Montmacq, les trois sépultures sont alignées est-ouest et distantes entre elles de moins de $5 \mathrm{~m}$. Elles appartiennent potentiellement au même groupe familial et sont inscrites dans l'espace domestique. À Pommiers, la sépulture, en limite de secteur, est interprétée comme possible marqueur territorial ; à Couloisy, le dépôt est secondaire et relégué dans une zone secondaire de type arrière-cour ; à Plailly, les deux sépultures d'immatures datées des $\mathrm{VII}^{\mathrm{e}}$-VIII ${ }^{\mathrm{e}}$ siècles sont localisées en marge de l'habitat, mais leur positionnement en limite d'emprise de la fouille ne permet pas d'affirmer leur caractère isolé. Les ensembles funéraires sont attestés sur cinq gisements : Mercin-et-Vaux ( $\left.{ }^{\circ} 5\right)$, ChâteauThierry $\left(n^{\circ} 8\right)$, Oudeuil ( $\left.n^{\circ} 18\right)$, Harly $\left(n^{\circ} 22\right)$ et Longueil-Annel ( $\left.n^{\circ} 24\right)$. Bien que les études soient en cours, on perçoit quelques caractéristiques exploitables dessinant au moins quatre morphologies particulières : à Mercin-et-Vaux, le cimetière, topographiquement lié à l'église, est abandonné au XII ${ }^{\mathrm{e}}$ siècle, phase de restructuration de l'espace interne villageois. À Château-Thierry, au « 3-5, rue de la Madelaine », la nécropole est abandonnée : du fait de l'évolution urbaine, elle est déplacée vers un faubourg de la ville voisine, en dehors de l'enceinte urbaine. À Harly, la localisation des sépultures semble en partie s'organiser le long d'un chemin
- devenu limite cadastrale contemporaine - en direction de l'église actuelle (communication orale d'Estelle Pinard, Inrap). Concernant l'habitat limitrophe, entraperçu lors du diagnostic, on en retrouve certains indices carolingiens dans les sépultures. Le traitement de ces données permettra peut-être de déterminer si la nécropole a continué ou non de fonctionner pendant la phase carolingienne de l'habitat. Enfin à Longueil-Annel, la nécropole est pérenne $\mathrm{du} \mathrm{V}^{\mathrm{e}}$ au Xi $\mathrm{XI}^{\mathrm{e}}$ siècle. Elle est installée sur la rupture sommitale d'un versant surplombant le village (Fréville, Journa, 1994). Dans le courant du IX ${ }^{\mathrm{e}}$ siècle, l'espace sépulcral est déplacé entre l'habitat et la rivière, en un lieu où sera érigée une église en pierre au Moyen Âge central, ce qui suggère l'hypothèse d'un édifice antérieur en bois. Ces différentes morphologies sépulcrales expriment quasiment toutes cette caractéristique principale des $\mathrm{VI}^{\mathrm{e}}-\mathrm{XI}^{\mathrm{e}}$ siècles, à savoir la cohabitation des morts et des vivants.

\section{De la création... à l'abandon ?}

Les phases de création des habitats paraissent nettement prédominantes aux $\mathrm{VI}^{\mathrm{e}}-\mathrm{VII}^{\mathrm{e}}$ siècles $(18$ cas). Huit d'entre eux perdurent jusqu'au XI ${ }^{\mathrm{e}}$ siècle voire le début du XII ${ }^{\mathrm{e}}$ siècle, quatre ont une durée de vie de trois siècles, les six derniers sont en cours de détermination. Pour les phases intermédiaires, $\mathrm{VII}^{\mathrm{e}}$ et $\mathrm{VIII}^{\mathrm{e}}$ siècles, les créations sont moins denses avec cinq cas potentiels. Pour deux d'entre eux, « le Chemin de la Patte d'Oie » ( $\left.n^{\circ} 30\right)$ et « le Chemin du Châtelain » $\left(\mathrm{n}^{\circ} 2\right)$, les données en cours d'étude laissent transparaître l'image d'une «fréquentation » des lieux pendant la phase II d'Édith Peytremann $\left(\mathrm{VI}^{\mathrm{e}}-\mathrm{mi} \mathrm{VII}^{\mathrm{e}}\right.$ siècle) avant une installation domestique avérée au VIII ${ }^{\mathrm{e}}$ siècle. Pour le $\mathrm{IX}^{\mathrm{e}}$ siècle (phase V Peytremann), trois cas sont à signaler : Lassigny $\left(n^{\circ} 27\right)$, Achery $\left(n^{\circ} 28\right)$ et Chevrières $\left(n^{\circ} 31\right)^{4}$. On ajoutera également le gisement de Passel $\left(n^{\circ} 3\right)$, dont la phase de création se situe entre le $\mathrm{IX}^{\mathrm{e}}$ et le $\mathrm{X}^{\mathrm{e}}$ siècle. En ce qui concerne la phase précoce (phase I Peytremann : IV $\mathrm{V}^{\mathrm{e}}-\mathrm{V}^{\mathrm{e}}$ siècles), seul le cas de Blesmes ( $\left.n^{\circ} 9\right)$ est mentionné. Cette opération, en périphérie du village, a mis au jour des traces d'occupation antique (villa et atelier de potier). Sébastien Ziegler avance l'hypothèse d'une évolution probable de l'habitat sur les vestiges d'une villa et d'une voirie antiques. Les quelques structures domestiques (parcellaire, enclos, trois « fonds de cabane ») sont abandonnées au $\mathrm{V}^{\mathrm{e}}$ siècle, l'habitat se déplaçant hors d'un secteur inondable. Ainsi, les $\mathrm{VI}^{\mathrm{e}}-\mathrm{VII}^{\mathrm{e}}$ siècles sont propices à l'émergence des installations au sein de notre corpus (18 cas sur 31) et font écho aux caractéristiques chronotopographiques relevées pour la Picardie (Peytremann, 2003, p. 165). A contrario, le deuxième pic de création identifié pour le $\mathrm{IX}^{\mathrm{e}}$ siècle par cette chercheuse est dans notre cas moins marqué. Le « pourquoi » de l'installation reste flou : raisons économiques? politiques? milieu géographique favorable? fait du hasard ? Certains gisements comme ceux de Mercin-et-Vaux $\left(n^{\circ} 5\right)$ et Pommiers $\left(n^{\circ} 6\right)$ semblent
4. Létude céramique

cours doit établir la

occupation

mérovingienne des lieux, pressentie lors du

diagnostic. 
exprimer la volonté de s'établir au pied du plateau du fait des potentialités de ressources maximales, climatiques et géologiques (plateau, coteau, fond de vallée). D'autres comme Montmacq $\left(\mathrm{n}^{\circ} 1\right)$ et Crécy-sur-Serre $\left(n^{\circ} 19\right)$ ont, en plus des ressources exploitables proches, la particularité de se «trouver » à proximité d'un palatium. Bien que le gisement de «la Croix Noire» $\left(\mathrm{n}^{\circ}{ }_{1}\right)$ ne concerne qu'une partie de l'habitat carolingien, la position orthonormée des structures suggère une création/ transformation selon un plan déterminé, donc une volonté politique. Concernant les phases d'abandon, les résultats actuels des 21 opérations de fouilles montrent un abandon peu marqué pour la période mérovingienne et un pic d'abandons/ désertions $\mathrm{au} \mathrm{XII}^{\mathrm{e}}$ siècle, phénomène déjà mis en évidence (Peytremann, 2003, p. 273). Même pic de désertion, donc, mais morphologie différente : on ne déserte pas de la même façon ni pour les mêmes raisons. Une première forme de désertion consiste en l'intégration de l'habitat rural en faubourg. À Chambly $\left(n^{\circ} 11\right)$ et à Château-Thierry $\left(n^{\circ} 8\right)$, la structure domestique et agricole de l'habitat ne résiste pas à la croissance urbaine. Une deuxième forme de désertion répond à l'installation d'une autorité seigneuriale caractérisée par l'implantation d'un château et une réorganisation du territoire, à l'exemple de Béhéricourt $\left(n^{\circ} 4\right)$. La place forte est fondée vers le XII ${ }^{\mathrm{e}}$ siècle sur un parcellaire préexistant recelant une occupation à vocation agricole et artisanale. C'est aussi le cas à Nogentl'Artaud qui est occupé dès le viI ${ }^{\mathrm{e}}$ siècle et cité dans le polyptique d'Irminon au IX ${ }^{\mathrm{e}}$ siècle. L'occupation domestique y est supplantée par un complexe castral à la fin du XII ${ }^{\mathrm{e}}$ siècle. Une troisième forme est identifiée, de loin la plus fréquente, celle du « glissement » vers l'espace qui deviendra le centrebourg actuel. Dans les cas de Pommiers $\left(n^{\circ} 6\right)$ et de Mercin-et-Vaux $\left(\mathrm{n}^{\circ} 5\right)$, un « recentrage » s'effectue vers ou autour de l'église. Dans le cas de Couloisy $\left(\mathrm{n}^{\circ} 10\right)$, le déplacement s'effectue vers le réseau viaire majeur à proximité. L'influence du milieu naturel est aussi invoquée, notamment à Passel $\left(n^{\circ} 3\right)$ où les berges de la rivière sont fréquentées au haut Moyen Âge, puis habitées à partir des $\mathrm{X}^{\mathrm{e}}-\mathrm{XI}^{\mathrm{e}}$ siècles. L'abandon est alors lié à la progression de la zone humide, mais également à la dépression démographique du XIV ${ }^{\mathrm{e}}$ siècle cette sortie de zone inondable se retrouve également sur le gisement de Couloisy $\left(\mathrm{n}^{\circ}{ }_{10}\right)$. On pourrait continuer la liste de ces cas particuliers d'abandons : Précy-sur-Oise et cet habitat du « Martray » $\left(\mathrm{n}^{\circ} 15\right)$ qui ne dépasse pas le statut de hameau satellite et qui, après une période de remise en culture des terres, se réactive en hameau ou faubourg de Précy, avec une nouvelle forme de voie et de parcellaire en lanières, avant de muer en ferme à cour fermée à l'époque moderne. À Villers-sous-Saint-Leu, les crues du ru et la succession des incendies, dont on ignore s'ils sont liés aux activités artisanales métallurgiques ou aux divers passages des bandes armées, semblent avoir raison de l'occupation.
Désertion ou abandon? Recentrage ou glissement? Les réponses sont multiples et modulables en fonction des particularismes locaux.

Du statut des habitats et de la question du " village »

Quel est donc le statut de ces habitats ? Lorsque la surface explorée a permis l'identification d'activités et/ou de fonctions économiques, il est envisageable de les « hiérarchiser ». La fonction domestique et/ou domestique agricole/pastorale est la forme dominante ( 25 sur 31 occurrences) au sein du corpus. Sur cette dominante se greffent quelques "particularismes » : deux gisements situés aux portes des villes, « Rue Henri Barbusse » à Chambly $\left(n^{\circ} 11\right)$ et « la Fontaine Saint-François » à Noyon $\left(\mathrm{n}^{\circ} 3\right)$ ont potentiellement pris une forme d'« habitat-greniers » dont les provisions de grains étaient destinés à la ville limitrophe. Les gisements de Montmacq $\left(n^{\circ} 1\right)$, Villers-sous-Saint-Leu $\left(n^{\circ} 13\right)$, Montigny-Lengrain ( $n^{\circ} 21$ ), Villers-Cotterêts $\left(n^{\circ} 25\right)$ déclinent au moins une activité artisanale en lien avec la pratique de la métallurgie du fer, de l'artisanat du textile ou du travail du cuir. Pour deux d'entre eux, Villers-Cotterêts et Villers-sousSaint-Leu, l'orientation artisanale s'avère primordiale : ces deux occupations s'apparentent à un « hameau » de forgerons, le gisement de la « Rue de Boissy 1 » $\left(\mathrm{n}^{\circ} 13\right)$ présentant le visage d'un habitat dédié à la forge, dont l'évolution morphologique se suit du $\mathrm{VI}^{\mathrm{e}}$ au XIII ${ }^{\mathrm{e}}$ siècle [cf. encadré p. 65]. Le gisement de « la Croix Noire » $\left(n^{\circ} 1\right)$ a livré quant à lui une quantité importante de mobiliers métalliques (couteaux, rejets scoriés...), de polissoirs, de tabletterie (poinçons, aiguilles...) ; les premières répartitions spatiales en cours laissent entrevoir à la période carolingienne une possible spécialisation des espaces internes et une production à visée commerciale tournée vers l'extérieur et les cités proches de Compiègne ou de Noyon (?). On pressent, au travers de ces différents cas, la trame d'une hiérarchie et les relations potentielles entre les différents types d'habitats, qu'ils soient modestes (domestique et/ou agricole), intermédiaires (domestique et/ou agricole et/ou artisanal), ou privilégiés (spécialisation des activités/circuit économique/présence politique). Mais là s'arrête l'essai de « hiérarchisation » en l'attente des résultats définitifs de l'ensemble des opérations pour clarifier cette ébauche. Car cette hiérarchisation effective nous permettra de participer « cartes en main » au débat villageois, débat encore prégnant aujourd'hui. D'autant que cette question n'a jamais été véritablement abordée de façon synthétique dans notre espace d'étude. Lors de la première réunion constitutive de l'ARC, le tour de table sur la notion « village » avait tourné court. Non par opposition mais plutôt par consensus : « avant de jouter, le chevalier doit s'assurer de sa monture et de ses armes ». Ainsi, les efforts doivent être portés, entre autres, sur l'analyse des sites, sur l'établissement des typologies structurelles, sur la confrontation des données de 
terrain et des sources anciennes, sur l'appréhension des données environnementales des territoires avant une quelconque prise de position « villageoise».

Bien que contraint par son échantillonnage restreint, son hétérogénéité quant à l'aboutissement des travaux de post-fouille, ses datations céramiques parfois imprécises du fait de l'indigence de certains ensembles collectés et de la documentation publiée, notre corpus dévoile malgré tout quelques facettes de son « potentiel». On notera, pour les gisements « exploités », l'importance relative du substrat antique, la prééminence des zones de vallées, l'omniprésence des caractères hydrographiques sur le site ou à proximité, le passage d'un habitat lâche mérovingien à un habitat groupé carolingien, la prédominance du pic de création lors des $\mathrm{VI}^{\mathrm{e}}$ $\mathrm{vII}^{\mathrm{e}}$ siècles, pour ne citer qu'eux. Caractéristiques que l'on retrouve tout ou partie dans les sphères d'études franciliennes, et à certain égard dans la partie nord-ouest de la France. D'autres thèmes émergent qui méritent de s'appesantir sur la question, comme le rôle structurant des voies de communications anciennes - où le carrefour, ancien ou créé ex nihilo, devient facteur de genèse ou fixation-, comme la question du « recentrage », $\mathrm{du}$ « glissement » vers les centres-bourg actuels dont l'église peut être une modalité mais ne doit plus être considérée comme l'unique vecteur de recentrage -, avec des glissements qui varient de quelques dizaines de mètres à parfois $500 \mathrm{~m}$. C'est un fait, la question du village, du « fait villageois », mais avant tout de l'habitat rural dans notre espace d'études accuse un retard certain au niveau des propositions de synthèses. On reste actuellement encore largement dépendant de travaux anciens (Bayard, 1986), de synthèses universitaires (Peytremann, 2003), de la vision « du village de Marle ». La dynamique amorcée récemment permettra, nous l'espérons, d'affiner certaines propositions, d'autant que le corpus commence à ne pas être négligeable : en associant toutes les données acquises de 1970 à 2014, celui-ci dépasserait la soixantaine de sites exploitables. Ce travail de reprise de corpus n'est pas le seul à engager : à l'imitation des autres régions à la pointe de la recherche, il convient de poursuivre la surveillance de tous les travaux, même limités à de petites surfaces, dans les villages actuels et en périphérie proche, et encore plus dans les communes déjà explorées. Les exemples de HauteYutz (Moselle), Serris (Seine-et-Marne), Tremblayen-France (Seine-Saint-Denis), Villiers-le-Sec (Val-d'Oise), Vitry-sur-Orne (Moselle) sont significatifs (Blaising, Gérard, 2006 ; Gentili, 2010 ; Gérard, 2012 ; Gonçalves-Buissart et al., 2012). Il faudrait également développer les travaux sur l'environnement pour mieux essayer de comprendre le paysage : il est donc essentiel de disposer d'équipes pluridisciplinaires, ce que projette d'établir cet axe de recherche (Catteddu, 2004). Que dire des traditions de datations? Des écueils liés aux effets de sources? Du manque de confrontation entre archives du sol et archives anciennes? Nani gigantum humeris insidentes: voilà ce par quoi le chemin commence.

\section{Références bibliographiques}

BAYARD D., 1986, « Le peuplement et l'habitat rural en Picardie à l'époque mérovingienne ", in La Picardie, berceau de la France: Clovis et les derniers Romains, 1500 anniversaire de la bataille de Soissons, 4861986, Catalogue de l'exposition, Amiens, Société archéologique de Picardie, p. 204-211.

BAYARD D., 1991, « Un village du temps d'Hugues Capet (987-996) à Longueil-Sainte-Marie », in Archéologie de la vallée de l'Oise. Compiègne et sa région depuis les origines, Catalogue d'exposition (Compiègne, 1991) Compiègne, Centre de recherche archéologique de la vallée de l'Oise, p. 183-184.

Blaising J.-M., GÉRARD F., 2006, « Les apports des opérations archéologiques de grandes surfaces aux connaissances du milieu rural médiéval et postmédiéval du nord lorrain ", in BRUN P., MARCIGNY C., VANMOERKerKe J., Une archéologie des réseaux locaux. Quelles surfaces étudier pour quelle représentativité?, Actes de la table ronde de Châlonsen-Champagne, 14-15 juin 2005, Les Nouvelles de l'Archéologie n ${ }^{\circ}$ 104-105, Paris, p. 22-28.

Blary F., Derbois M., Legros V., 2005, « Les campagnes médiévales en Picardie : État de la question », in La Recherche archéologique en Picardie: Bilans et perspectives, Actes des Journées d'études tenues à Amiens les 21 et 22 mars 2005 , Revue Archéologique de Picardie 3/4, p. 223-246.

Cattedu I., 2004, « Archéologie préventive et analyse des occupations rurales durant le haut Moyen Âge ", Archéopages, 13, juillet 2004, p. 26-31.

Desplanque G., 2013, Pommiers «Les Grosses Vignes », rapport de diagnostic, Conseil général de l'Aisne, $33 \mathrm{p}$.

Desplanque G. et al., 2014, Pommiers « Les Grosses Vignes » (Aisne), rapport final d'opération, Conseil général de l'Aisne, 2 vol., $150 \mathrm{p}$.

DEVROEY J.-P., 2003, Économie rurale et société dans l'Europe franque ( $V I^{e}-I X^{e}$ siècle), t. 1, Fondements matériels, échanges et lien social, Paris, Belin, $380 \mathrm{p}$.
Fréville C., Journa R., 1994, « La nécropole mérovingienne de Longueil-Annel (Oise) ", Revue archéologique de Picardie, 1994, p. 87-178.

Fronty R. et al., 2013, Oudeuil, Oise, Rue Saint-Omer rapport de diagnostic, Inrap-SRA Picardie, $54 \mathrm{p}$.

GENTILI F., 2010, «L'organisation spatiale des habitats ruraux du haut Moyen Âge : l'apport des grandes fouilles préventives. Deux exemples franciliens : Serris « les Ruelles » (Seine-et-Marne) et Villiers-leSec (Val-d'Oise) », in CHAPELOT J. (DIR.), Trente ans d'archéologie médiévale en France. Un bilan pour un avenir, Caen, Publications du CRAHM, p. 119-131.

GÉRARD F., 2012, « La structuration du village pour une économie agraire planifiée à la fin du $\mathrm{IX}^{\mathrm{e}}$ siècle en Lorraine. Les sites de Vitry-sur-Orne et Demangeaux-Eaux », Archéopages, 34, juillet 2012, p. 38-47.

Goncalves-Buissart C., Lafarge I., Le Forestier C. 2012, "Les habitats ruraux du haut Moyen Âge en Seine-Saint-Denis. État des lieux ", Archéopages, 34 , juillet 2012, p. 48-57.

JAKUBOWSKI J.-F., 2000 : « Chevrières, église SaintGeorges (Oise) ", in Bilan Scientifique de Picardie 1998, Amiens, SRA de Picardie, p. 62.

JouANIN G., 2014, «Étude archéozoologique du site de Pommiers "Les Grosses Vignes". Périodes mérovingienne et carolingienne », in DESPLANQUE G. et al., Pommiers «Les Grosses Vignes » (Aisne), rapport final d'opération, Conseil général del'Aisne, vol. 1,152 p.

Lebègue M., 1994, Les Noms des communes du département de l'Oise, Amiens, Musée de Picardie, 234 p.

LorReN C., PÉRIN P. (DIR.), 1995, L'Habitat rural du haut Moyen Âge (France, Pays-Bas, Danemark et Grande-Bretagne), Actes des XIV's journées internationales d'archéologie mérovingienne Guiry-en-Vexin et Paris, 4-8 février 1993, Rouen, Association française d'archéologie mérovingienne, Mémoires de l'AFAM, t. VI, XIV-237 p.
MARTIN J.-F. (DIR.) et al., 2011, Le site de "Jeoffrécourt" à Sissonne (Aisne), cimetière et unités domestiques $d u I^{e}$ au IX $X^{e}$ siècle après J.-C., Revue archéologique de Picardie, 1/2, 2011, 394 p.

Pecqueur L., 2003 : « Des morts chez les vivants : les inhumations dans les habitats ruraux du haut Moyen Âge en Île-de-France ", Archéologie médiévale, 33, p. 1-31.

Peytremann É., 2003, Archéologie de l'habitat rural dans le nord de la France du IV $V^{e}$ au XII siècle, Saint-Germain-en-Laye, Association française d'archéologie mérovingienne, Mémoires de l'AFAM, t. XIII, 2 vol., 453-44,2 p.

Pissot V., Bruant J., Cotté O., Desrayaud G., Gautier S., Hénon B., Hugonnier L., 2013, « Vestiges de bornage antique en Île-de-France et en Picardie. La question des dépôts de vases dans les fossés ", Archéopages, 36, janvier 2013, p. 58-69.

Querrien A., Bui Thi MAÏ, Girard M., 2006, « La pertinence des analyses palynologiques pour la reconstitution des paysages médiévaux ", in L'Archéologie médiévale en France depuis 30 ans, Les dossiers d'archéologie, 314 , juin 2006, p. 14-17.

ROBERT S., 2009, « L'archéologie des voies aujourd'hui. Un renouveau par l'archéogéographie et l'archéologie préventive ", Archéopages, 27, octobre 2009, p. 54-57.

Thouvenot S., 2010, Pommiers (Aisne), « Les Grosses Vignes ». Un habitat du premier Moyen Âge, rapport de diagnostic, Inrap-SRA Picardie, $42 \mathrm{p}$.

TREFFORT C.,1996, L'Église carolingienne et la Mort. Christianisme, rites funéraires et pratiques commémoratives, Lyon, Presses universitaires de Lyon, Collection d'Histoire et d'Archéologie médiévales, 3, 219p.

VAlais A. (dir.), 2012, L'Habitat rural au Moyen Âge dans le nord-ouest de la France, t. 1, Les synthèses, Rennes, PUR, 328 p. 